\title{
TiO and $\mathrm{H}_{2} \mathrm{O}$ absorption lines in cool stellar atmospheres
}

\author{
France Allard \\ C.R.A.L (UML 5574) Ecole Normale Superieure, 69364 Lyon Cedex 7, rance \\ E-Mail: fallard@ens-lyon.fr \\ Peter H. Hauschildt \\ Dept. of Physics and Astronomy E Center for Simulational Physics, University of Georgia, \\ Athens, GA 30602-2451 \\ Email: yeti@hal.physast.uga.edu \\ and \\ David Schwenke \\ NASA Ames Research Center, Mail Stop 230-3, Moffett Field, CA 94035-1000 \\ E-mail: schwenke@pegasus.arc.nasa.gov \\ ApJ, in press. Also available at \\ ftp://calvin.physast.uga.edu/pub/preprints
}

\section{Introduction}

Over the past decade, model atmospheres and synthetic spectra for late-type stars have improved hand-in-hand with higher quality opacities. In 1994, quality lists of transitions of the water vapor molecule based on ab initio molecular calculations became available (Miller et al. 1994; Schryber, Miller, \& Tennyson 1995; Jørgensen, Jensen, \& Sørensen 1994) which allowed the computation of the first Direct Opacity Sampling (hereafter dOS) model atmospheres for late-type dwarfs (Allard et al. 1994) and brown dwarfs (Allard et al. 1996), later to become the NextGen models described in (Hauschildt, Allard, \& Baron 1999). Showing a more physical description of their main opacities, the NextGen dOS model atmospheres promised a better description of the Spectral Energy Distribution (hereafter SED) of cool stars. And this appeared to be verified for the infrared SED of M dwarfs (Jones et al. 1996; Leggett et al. 1996; Allard et al. 1997).

But despite these fundamental improvements, the NextGen models have failed to match adequately several of the optical (spectroscopic and photometric) properties of late type dwarfs and giants. In fact, the dOS models (Brett 1995; Allard et al. 1994, 1997; Hauschildt, Allard, \& Baron 1999) provided a worse fit to the optical SED of lower main 
sequence dwarfs than previous models based on simplified molecular opacities (Allard \& Hauschildt 1995, hereafter, AH95). The models could not reproduce the tight relation formed by $\mathrm{M}$ dwarfs in the V-R versus R-I two-color diagram, indicating a systematically shallower slope of the optical SED (defined by TiO absorption) then observed in these stars. A systematic flux excess in the spectral region sampled by the $\mathrm{V}$ bandpass $(0.4-0.65 \mu \mathrm{m})$ was noted as well in dwarfs as in red giants. Baraffe et al. (1998a) observed that this excess in the NextGen dwarf models translated into lower main sequence isochrones deviating progressively to the blue (by up to 1.0 magnitude!) in ( $\left.\mathrm{M}_{V}, \mathrm{~V}-\mathrm{I}\right)$ colormagnitude diagrams, for masses lower than about $0.5 \mathrm{M}_{\odot}\left(\mathrm{T}_{\text {eff }} \leq 3800 \mathrm{~K}\right)$. Baraffe et al. (1997a) examined a variety of globular clusters and showed that this departure of the models decreased in amplitude with decreasing metallicity. The problem seemed therefore confirmed to be caused by a lack of opacity of an oxygen compound ${ }^{1}$.

Only three independent models of the $\mathrm{TiO}$ molecule and corresponding lists of transitions were available so far to the construction of model atmospheres. The first model was constructed over two decades ago by Collins (1975) and was restricted by the computational limitations of the time. The Collins line list was intended to model the extended atmospheres of red giants and did not include high energy and otherwise weak transitions important by their number in the hotter environments of red dwarf atmospheres. It also neglected the red $\epsilon$ system of TiO. Jørgensen (1994) extended Collins' work to TiO isotopic transitions, included the $\epsilon$ system from revised molecular rotational constants, and adopted the laboratory oscillator strengths of Davis, Littleton, \& Phillips (1986). It is therefore understandable that the resulting limited list of transitions caused shortcomings in the NextGen model atmospheres. The second TiO list was constructed by Kurucz (1993) and is used in his ATLAS9-12 atmospheres. The third model was constructed by Plez (1992) using also the Davis, Littleton, \& Phillips (1986) oscillator strengths, and is used in his version of the MARCS atmosphere code. All three independent models yielded the visual flux excess in different proportions. Plez (1998) suggested that the missing opacity is due to missing $\mathrm{TiO}$ band systems in current lists, and added the $\mathrm{TiO}$ a-f system at 0.5 $\mu \mathrm{m}$ to his list. However, this helped him only partially to resolve the V-band flux excess problem.

Recently, Langhoff (1997) constructed a new model of the TiO molecule and published new lifetimes and oscillator strengths that improved significantly upon the 1986 values of Davis et al. (Valenti, Piskunov, \& Johns-Krull 1998; Plez 1998). Schwenke (1998) has subsequently computed a corresponding list of transitions complete to the high ener-

\footnotetext{
${ }^{1}$ Hydride absorption bands only get stronger relative to the continuum with decreasing metallicity over the range covered by the globular clusters studied in Baraffe et al. (1997a) in the optical spectral range.
} 
gies and therefore more suitable for general model atmosphere applications. In this paper we present the results of including this new $\mathrm{TiO}$ line list as well as the new AMES- $\mathrm{H}_{2} \mathrm{O}$ list in the construction of model atmospheres and synthetic spectra for late-type dwarfs and red giants.

\section{Model calculations}

We have calculated the models presented in this paper using version 10.3 of our general model atmosphere code PHOENIX. Details of the code and the general input physics are discussed in Hauschildt, Allard, \& Baron (1999) and references cited therein. The models for $\mathrm{M}$ giants were calculated with the same setup, however, they employ spherical geometry (including spherically symmetric radiative transfer). For giant models with low gravities $(\log (g) \leq 3.5)$, this can be an important effect for the correct calculation of the structure of the model atmosphere and the synthetic spectrum (Aufdenberg et al. 1998, 1999). The main difference between the models presented in Hauschildt, Allard, \& Baron (1999) and the models presented here is the use of the new AMES line lists for $\mathrm{H}_{2} \mathrm{O}$ (Partridge \& Schwenke 1997) and TiO (Schwenke 1998), but we have also adjusted the empirical oscillator strengths of $\mathrm{VO}$ and $\mathrm{CaH}$ absorption bands to respect their strength relative to $\mathrm{TiO}$ bands (note that $\mathrm{VO}$ and $\mathrm{CaH}$ absorption is still treated in the Just Overlapping Line Approximation due to lack of adequate line data). Our combined molecular line list includes about 500 million molecular lines. These lines are treated with a dOS technique where each line has its individual Voigt (for strong lines) or Gauss (weak lines) line profile (in the standard OS method tables of precomputed opacities are used). They are selected for every model from the master line list to the beginning of each model iteration to account for changes in the model structure, see Hauschildt, Allard, \& Baron (1999) for details. This procedure selects about 215 million molecular lines for a typical

giant model with $T_{\text {eff }} \approx 3000 \mathrm{~K}$ and about 130 million molecular lines for a dwarf model with the same effective temperature. Therefore, we generally use the parallelized version of phoenix (Hauschildt, Baron, \& Allard 1997; Baron \& Hauschildt 1998; Hauschildt \& Baron 1999) to perform the calculation efficiently on parallel supercomputers. Details of the $\mathrm{TiO}$ and $\mathrm{H}_{2} \mathrm{O}$ lists are given in the next subsections.

\subsection{Water lines}

The effects of water lines on the M dwarfs SED was discussed in Allard et al. (1994). For the work presented here, we have replaced the UCL water vapor line-list (Miller et al. 
1994; Schryber, Miller, \& Tennyson 1995, hereafter: MT- $\mathrm{H}_{2} \mathrm{O}$ ) used in Hauschildt, Allard, \& Baron (1999) with the AMES water line-list (Partridge \& Schwenke 1997, hereafter: AMES- $\mathrm{H}_{2} \mathrm{O}$ ). This list includes about 307 million lines of water vapor. For the calculations shown in this paper we have used $\mathrm{H}_{2}^{16} \mathrm{O}$ and neglected other, much less abundant, isotopes of this molecule.

The water vapor opacity is governed by the completeness of the line list used, but also by the adopted atomization energy. The partition function of the molecule cancels out in the final absorption coefficient, after we have multiplied cross-sections by number densities. But since water is an important chemical equilibrium specie, errors in the partition function can affect indirectly the model structure and spectra. The AH95 models were based on the Ludwig (1971) hot flames water cross-sections in the form of straight means, and used the JANAF partition function for water vapor (Irwin 1988). The NextGen models where, on the other hand, computed with the MT- $\mathrm{H}_{2} \mathrm{O}$ line list and a partition function computed from the MT- $\mathrm{H}_{2} \mathrm{O}$ levels. We note that the AMES- $\mathrm{H}_{2} \mathrm{O}$ partition function is practically identical to JANAF values, while the $\mathrm{MT}-\mathrm{H}_{2} \mathrm{O}$ value is smaller than JANAF for temperatures above $3000 \mathrm{~K}$, possibly due to the energy levels missing in the $\mathrm{MT}-\mathrm{H}_{2} \mathrm{O}$ data. We have therefore adopted for this and later work the JANAF partition function. We use an atomization energy of $9.5119 \mathrm{eV}$ from Irwin (1988) for all models since AH95.

\subsection{TiO lines}

The main point of this paper is the comparison of the model structure and the synthetic spectra obtained by using the list of $\mathrm{TiO}$ lines from (Jørgensen 1994, hereafter: SCAN-TiO) and the new list of TiO lines from (Schwenke 1998, hereafter, AMES-TiO). The AMES-TiO list includes a total of about 172 million lines, about 44.6 million of these are for the most abundant isotope ${ }^{48} \mathrm{TiO}$ and about 32 million lines for each of the remaining 4 isotopes ${ }^{46,47,49,50} \mathrm{TiO}$ ). But beyond the completeness of the line-list, two more considerations affects the overall opacity produced by $\mathrm{TiO}$, and explain systematic differences between model versions and by different authors: the atomization energy $\left(D_{0}^{0}\right)$ determines the number density of $\mathrm{TiO}$, and the $\mathrm{TiO}$ band oscillator strength ${ }^{2}$ have been derived from sunspot observations (Davis, Littleton, \& Phillips 1986, hereafter: DLP86), laboratory experiments (Hedgecock, Naulin, \& Costes 1995, hereafter: HNC95), as well as from ab initio calculations (Langhoff 1997, hereafter L97). (Brett 1990, hereafter:B90)

\footnotetext{
${ }^{2} \mathrm{f}_{\mathrm{el}}=f_{\nu^{\prime} \nu^{\prime \prime}} / q_{\nu^{\prime} \nu^{\prime \prime}}$, where the $f^{\prime} \mathrm{s}$ are the oscillator strengths and the $q^{\prime}$ s are the Franck-Condon factors of the transition $\nu^{\prime} \nu^{\prime \prime}$
} 
derived astrophysical $\mathrm{f}_{\mathrm{el}}$ values by fitting the optical SEDs of red giants, using an atomization energy of $7.76 \mathrm{eV}$. He quoted that reducing this value by $0.3 \mathrm{eV}$ would increase his $\mathrm{f}_{\mathrm{el}}$ by a factor 2.5. The most recent estimate of $\mathrm{D}_{0}^{0}$ for $\mathrm{TiO}$ is now $6.92 \mathrm{eV}$, which suggests that the $\mathrm{B} 90 \mathrm{f}_{\mathrm{el}}$ values are underestimated by as much as a factor 7 ! We summarize in table 1 the various sources of oscillator strengths available for $\mathrm{TiO}$.

The models of Allard (1990) used $\mathrm{f}_{\mathrm{el}}$ values from B90 together with the straight-mean TiO opacities by Collins (1975) and Collins \& Faÿ (1974), and assuming an atomization energy of $6.87 \mathrm{eV}$. The first comparison of these models to the SED of M dwarfs (Kirkpatrick et al. 1993) revealed the inadequacy of this combination of parameters for $\mathrm{TiO}$ which produced far too weak optical opacities. We have therefore, since the AH95 model series, employed the updated value of $6.92 \mathrm{eV}$, together with the larger laboratory $\mathrm{f}_{\mathrm{el}}$-values of DLP86. These two modifications combined to significantly increased the strength of $\mathrm{TiO}$ opacities in the models, bringing the AH95 and later the dOS NextGen models in improved agreement with the SED of M dwarfs. Any differences in the predictions of the AH95 and NextGen models are therefore purely due to the opacity technique (StraightMean versus dOS) and to the completeness of the line-list used. The incompleteness of the SCAN-TiO line-list allows photons to escape between absorption bands (see e.g. Valenti, Piskunov, \& Johns-Krull 1998), and thus leads to systematically and increasingly (with higher $T_{\text {eff }}$ ) bluer optical colors (V-I) than observed (Baraffe et al. 1998b, 1997b). For the current models we therefore explore the use of the more complete AMES-TiO line-list, and the yet larger theoretical $\mathrm{f}_{\mathrm{el}}$-values of L97.

\section{Results}

We have calculated a number of model atmospheres using either the SCAN-TiO or the AMES-TiO list of $\mathrm{TiO}$ lines and using either AMES- $\mathrm{H}_{2} \mathrm{O}$ or $\mathrm{MT}-\mathrm{H}_{2} \mathrm{O}$ as source of the $\mathrm{H}_{2} \mathrm{O}$ lines. All the other input physics is the same for both sets of models. All models have been fully converged with their respective set of parameters. Note that these models have been constructed for the purpose of this paper only and not to model individual stars and thus do not include dust formation and opacities which is important in atmosphere models with effective temperatures below about $2500 \mathrm{~K}$, such models are presented in a subsequent paper. In the following, we will discuss the results for the dwarf and giant models separately. The baseline for our comparisons are the NextGen models (Hauschildt, Allard, \& Baron 1999) for the dwarfs and the NG-giant models (Hauschildt et al. 1999) for the giants. 


\subsection{Effects of different TiO line lists}

The models discussed in this section were all calculated using AMES- $\mathrm{H}_{2} \mathrm{O}$ to isolate the effects of different $\mathrm{TiO}$ line lists on the model spectra and structures.

\subsubsection{M dwarf models}

In Figs. 1 and 2 we show a comparison of model spectra calculated with AMES$\mathrm{TiO}$ (full curves) and with SCAN-TiO (dotted curves) (both using our adopted $\mathrm{f}_{\mathrm{el}}$-set, as quoted in table 1$)$ for several effective temperatures. The gravity $(\log (g)=5.0)$ and abundances (solar) were selected to be representative of $\mathrm{M}$ dwarfs in the solar neighborhood. In both figures, the resolution of the synthetic spectra was reduced by boxcar smoothing to $20 \AA$. At high effective temperatures, the two sets of models are nearly identical due to reduced importance of $\mathrm{TiO}$ absorption. At very low $T_{\text {eff }}$ the two line lists apparently agree very well since only the lowest levels of $\mathrm{TiO}$ remain populated. It is essentially between $T_{\text {eff }} \approx 2000 \mathrm{~K}$ and $\approx 3500 \mathrm{~K}$ that the largest completeness and quality effects of the TiO line lists are seen.

Fig. 3 indicates the location of each $\mathrm{TiO}$ band system for a $2900 \mathrm{~K}$ model. From this it becomes clear that the addition of a-f transitions, which depress the continuum from 0.4 to $0.5 \mu \mathrm{m}$, is one of the largest improvements brought by the AMES-TiO list to our models. We note that the entire optical regime from 0.4 to $0.75 \mu \mathrm{m}$ shows generally more opacity in the AMES-TiO models then using the Jørgensen (1994) (hereafter: J94) line list. The $\epsilon$ bands at 0.82 to $0.88 \mu \mathrm{m}$ have a more precise shape in the AMES-TiO list, and come out stronger as well. This is a result of the completeness of the AMES-TiO list which also removes flux excess escaping between the troughs of the bands. We note however that some regions, such as in the $\gamma$ band near $0.78 \mu \mathrm{m}$, show less opacity in the new models.

The main effect of the new AMES-TiO on spectroscopic and photometric $T_{\text {eff }}$ estimates will however be dominated by the change we make to the oscillator strengths. The L97 $\mathrm{f}_{\mathrm{el}}$ values being generally smaller than the DLP86 values adopted by J94, models of early-type $\mathrm{M}$ dwarfs using the new AMES-TiO setup should predict systematically lower effective temperatures then did prior models (NextGen, AH95, etc., see also Fig.10 below). And beyond the enhanced completeness of the AMES-TiO list to high temperature transitions, the need for a cooler model should also contribute to making the TiO bands fit better a given star i.e. larger bands with less flux escaping from deeper, hotter atmospheric regions between them. 
We could have opted to use the HNC95 laboratory $f_{\mathrm{el}}$-values as did Plez (1998), but since the L97 ab initio values agree quite well, we decided to keep these, except for the $\delta$ and $\varphi$ band systems. The reason the oscillator strengths for the $\delta$ and $\varphi$ bands are less accurate is that it is very hard to get a good description of the $b$ state, which is the upper state in both bands. For the $\delta$ system, L97 derives an oscillator strength which is, as opposed to all other bands, twice as large as the DLP86 value. And the $\delta$ and $\varphi \mathrm{f}_{\mathrm{el}}$-values cannot be corroborated by recent experimental values. Such a strong $\delta$ band system would be difficult to bring in agreement with $\mathrm{M}$ dwarfs observations. Indeed, prior models have all shown a gradually increasing departure to the blue of the main sequence in $\mathrm{M}_{V}$ vs $V-I$ diagrams (Baraffe et al. 1995, 1998b). Such departure is significantly improved using the new $\mathrm{TiO}$ list if one keeps a weak $\delta$ band as indicate preliminary results of evolution models to be published separately (see also Fig. 12 below). We have therefore adopted to keep the DLP86 oscillator strength values for the reddest two TiO bands until new laboratory experiments can either confirm or infirm the L97 predictions. The summary of our adopted set of oscillator strengths for $\mathrm{TiO}$ is presented in Table 1.

\subsubsection{M giant models}

The results for the giant models are similar to the results for the dwarfs. Figures 4 and 5 show synthetic spectra for 3 representative giant models with the indicated effective temperatures. The models have in common the parameters $\log (g)=0.5, M=5 \mathrm{M}_{\odot}$ and solar abundances. The differences between the AMES-TiO (full curves) and SCAN-TiO (dotted curves) models are somewhat larger for giants than for the dwarfs in the blue spectral region due to an increased sensitivity to the added a-f system opacities in the AMES-TiO line list. It is however somewhat less pronounced in the red spectral region where $\mathrm{TiO}$ bands are weaker in giants. The "spikes" that are apparent in the SCAN-TiO spectrum with $T_{\text {eff }}=3000 \mathrm{~K}$ are absent in the AMES-TiO models. These spikes were one of our major problems in fitting observed spectra of giants. For larger $T_{\text {eff }}$ the differences between the spectra diminishes quickly as TiO becomes less important in the giants. This happens at lower effective temperatures compared to the dwarfs because of the lower pressures in giant atmosphere which results in smaller partial pressure of molecules as compared to dwarfs. 


\subsubsection{Model Structures}

A comparison of the model structures for both dwarf and giant model reveals only very small differences between structures calculated with AMES-TiO and SCAN-TiO. We plot the differences in electron temperature as well as the relative differences between the AMES-TiO and SCAN-TiO models for dwarfs and giants in Figs. 6 and 7, respectively. The changes are generally very small, only in the per-cent range for the gas pressures and about $10 \mathrm{~K}$ maximum difference between the electron temperatures for the dwarf models. For the giant models the differences are somewhat larger. The changes in the opacity averages are generally small but largest for the Rosseland mean opacity in the outer layers of the giant models. The temperatures are higher in AMES-TiO model for both the giant and the dwarf models, however, the gas pressures are lower in the AMES-TiO dwarf model but higher in the AMES-TiO giant model. Overall the changes are modestly small, indicating that the detailed effects of the $\mathrm{TiO}$ line lists do not have a large effect on the model structure itself.

\subsection{Effects of different water vapor line lists}

In figure 8 we show the effects of different water line lists on the synthetic spectra for $\mathrm{M}$ dwarfs. All models shown in the graph otherwise use the same line-lists (AMES-TiO was used for the $\mathrm{TiO}$ lines). In overall, we can see that the changes in the water vapor line lists are of larger amplitude than the changes in the completeness of the $\mathrm{TiO}$ line list. The models calculated with AMES- $\mathrm{H}_{2} \mathrm{O}$ show a totally different shape of the $1.4 \mu \mathrm{m}$ band, both weaker and wider then predicted by the MT- $\mathrm{H}_{2} \mathrm{O}$ model. The completeness of the new line list to high temperatures helps block more flux escaping from deeper, hotter layers of the models around $1.6 \mu \mathrm{m}$, and $2.2 \mu \mathrm{m}$. This promises a much better description of observations in general.

We also find important changes of the model structure as shown in Fig.9. The differences of the electron temperatures can reach $100 \mathrm{~K}$, the gas pressures can differ by $20 \%$ and the opacity averages, in particular the Rosseland mean, can differ by close to $60 \%$. These effects are much larger in the outskirts of the atmosphere than the changes in the structures caused by different $\mathrm{TiO}$ line lists (see Fig. 6). As a result, the use of the AMES$\mathrm{H}_{2} \mathrm{O}$ line list also affects the optical spectra, causing weaker $\mathrm{TiO}$ bands than obtained with the MT- $\mathrm{H}_{2} \mathrm{O}$ line list. Models of early-type $\mathrm{M}$ dwarfs based on the AMES- $\mathrm{H}_{2} \mathrm{O}$ line list therefore systematically predict yet lower effective temperatures for a given star. 


\subsection{Combined effects}

In Figs. 10 and 11 we display a comparison between NextGen models (which use MT- $\mathrm{H}_{2} \mathrm{O}$ and SCAN-TiO) and models that use the AMES- $\mathrm{H}_{2} \mathrm{O}$ and AMES-TiO. The wavelength range of important filters and band identifications for $\mathrm{TiO}$ are given on the figures. The $\mathrm{TiO}$ bands in the "AMES-atmosphere" are considerably weaker than those of the NextGen model spectrum as a result of the smaller oscillator strengths used, and the structural effects. On the other hand, the water bands are stronger in the AMESatmosphere than in the NextGen model. This model has a relatively high temperature, thus the higher energy levels of the water molecule are relatively more important than for models with lower $\mathrm{T}_{\text {eff }}$ (however, the concentration of water molecules is reduced so the overall water opacity is smaller than in the cooler models).

To better judge of the impact of these opacity changes on the overall SED of M dwarfs in general, we have computed synthetic photometry as described in AH95 for three sets of models: (1) the NextGen grid based on J94 and MT- $\mathrm{H}_{2} \mathrm{O}$ (2) the AMES grid based on AMES-TiO and AMES- $\mathrm{H}_{2} \mathrm{O}$ opacities, and (3) the AMES-MT grid based on AMES-TiO and $\mathrm{MT}-\mathrm{H}_{2} \mathrm{O}$ opacities. The results are compared to a photometric sample of $\mathrm{M}$ dwarfs (Leggett 1992) in Figs. 12, 13, and 14. Since M dwarfs form a tight sequence in optical VRI two-colors diagram despite the age and metallicity scatter of the sample (see AH95), this diagram imposes a strong constraint on model atmospheres. We find that models based on AMES-TiO opacities are systematically redder in $V-R$ and $V-I$ than models based on the J94 line list. The new models agree much better with observations and the new $\mathrm{TiO}$ data removes most of the discrepancy shown by the NextGen models in the lower main-sequence. Small remaining discrepancies may be attributed to the JOLA handling of $\mathrm{VO}$ and $\mathrm{CaH}$ which tend to overestimate slightly their opacities in the present models. Leinert et al. (1999) already studied the low resolution HST/FOS spectra of an M6 dwarf (LHS1070A) and found the AMES-MT models indeed agree quite well both with the observed SED and absolute fluxes within errors on the parallax of the system. They however noticed that some "continuum" flux excess remains important in the visual part of the SED $(0.45$ to $0.65 \mu \mathrm{m})$. However, there is no a priori reason to assume that the a-f oscillator strengths are inaccurate, and these remaining problems could be related to other effects on the model structure.

The use of AMES- $\mathrm{H}_{2} \mathrm{O}$ seems also to bring some improvements to the modeling of near-infrared colors. Fig. 13 shows that the late-type dwarfs can be better reproduced by the new water opacities than by the $\mathrm{MT}-\mathrm{H}_{2} \mathrm{O}$ line list. However this diagram is sensitive to both gravity (lower gravity models loop lower) and metallicity, which makes it difficult to constrain the models on the adequacy of the water opacities used with them. Leggett, 
Allard, \& Hauschildt (1998) and Leggett et al. (1999) have already used the AMES models in their analysis of $\mathrm{M}$ dwarfs and brown dwarfs, and found an excellent general agreement of the predicted near-infrared SED with observations. However these analyses used the models to derive the parameters of the studied stars and brown dwarfs based on fits to the near-infrared SED or photometry, and could not make an independent statement on the quality of the water line list.

$\mathrm{M} \mathrm{dwarfs} \mathrm{form} \mathrm{again} \mathrm{a} \mathrm{sequence} \mathrm{in} \mathrm{the} \mathrm{mixed-colors} \mathrm{IJK} \mathrm{diagram} \mathrm{(Fig.} \mathrm{14),} \mathrm{although}$ less tightly than in the VRI diagram. Unresolved binary stars produce K-band flux excess and lie below the sequence. $\mathrm{H}_{2}$ pressure-induced opacities depress the K-band flux of metal-depleted dwarfs so that they systematically lie above the sequence. But, as opposed to the JHK diagram, this one is not particularly sensitive to gravity in M dwarfs which allows a sequence to be defined. Models should pass therefore through the bulk of early-type $\mathrm{M}$ dwarfs at $J-K=0.8$, and follow a relatively $J-K$-insensitive sequence towards late-type dwarfs. We find that models based on the AMES- $\mathrm{H}_{2} \mathrm{O}$ opacities lie, as did the AH95 models before them, 0.2 magnitude in $J-K$ to the blue of the observed sequence! And our tests show that this result is independent of the TiO opacities used. The NextGen models already reproduced perfectly the location of lower main-sequence stars in this diagram. And AMES-MT models computed using the AMES-TiO and MT$\mathrm{H}_{2} \mathrm{O}$ line lists behave adequately both in the optical and infrared. Why? Perhaps the new water vapor line list is still not complete enough to high temperatures and lacks opacity in the J-bandpass i.e. around $1.3 \mu \mathrm{m}$ ? Or would it have too much opacity in the $K$-window i.e. around $2.2 \mu \mathrm{m}$ ? Until these questions can be answered, we hope that the two main grids of models we have computed (AMES and AMES-MT) will allow independent detailed confrontations to observations of cool stars that will locate more precisely the source of the problem (e.g. Leinert et al., in preparation).

\section{Summary and Conclusions}

A long standing problem with $\mathrm{M}$ dwarf models was that prior $\mathrm{TiO}$ line lists were incomplete to high temperatures. The use of "straight means" (AH95 models) helped by the coarseness of the treatment to block flux which otherwise escapes between lines in the incomplete list. But these models also blocked too much flux in most cases, and were only appropriate in late-type $\mathrm{M}$ dwarfs when $\mathrm{TiO}$ bands are already very strong. Clearly, a more complete line list was needed to model stars from the onset of $\mathrm{TiO}$ formation to its gradual disappearance from the gas phase in brown dwarfs. The AMES-TiO list now serves beautifully this purpose. We find that the list provides more opacity in most 
bands and suppress adequately flux between bands. The new, smaller oscillator strength values also play an important role in systematically assigning cooler models (at least for early type $\mathrm{M}$ dwarfs) to a given star, this way contributing to broader bands and lesser inter-band flux as well. These effects combine and should resolve most of the previously observed discrepancy between models and observations in the optical SED and photometry of M stars. Leinert et al. (1999) note however that flux excess remains substantial in the visual spectrum, suggesting some further incompleteness or $f_{e l}$ inaccuracies of the new $\mathrm{TiO}$ in the a-f system.

In order to better reproduce the observed (V-I) color indices, we had to retain in the present models the Davis, Littleton, \& Phillips (1986) $\mathrm{f}_{\mathrm{e}^{\mathrm{l}}}$-values for the two reddest band systems: $\delta$ and $\varphi$. For these two band systems, the theoretical estimates of Langhoff (1997) predicts an unexpectedly large $\mathrm{f}_{\mathrm{el}}$-value ratio, while no laboratory estimate (Hedgecock, Naulin, \& Costes 1995) are available to corroborate this. And we find, as did Alvarez \& Plez (1998) in red giants for the $\delta$ band, that models based upon the DLP86 $\mathrm{f}_{\mathrm{el}}$-values for these two bands reproduce adequately their observed depths in $\mathrm{M}$ dwarfs.

The introduction of the AMES- $\mathrm{H}_{2} \mathrm{O}$ opacities bring solid improvements of the nearinfrared SED of late-type dwarfs, but fails as the AH95 models did to reproduce adequately the $J-K$ colors of hotter stars. Water vapor is a more important factor for the structure of the atmosphere than $\mathrm{TiO}$ because its overall opacity is larger and its lines are closer to the peak of the SED than the TiO bands, so the flux blocking effect of water vapor is more important for the temperature structure than that of $\mathrm{TiO}$ opacities for these low temperatures. Schwenke and collaborators at NASA AMES are preparing a new dipole moment function for $\mathrm{H}_{2} \mathrm{O}$, which may change the high temperature high overtone water bands, and help resolve this discrepancy in the near future.

Until a revised version of the AMES- $\mathrm{H}_{2} \mathrm{O}$ line list becomes available, we have therefore generated two sets of model atmospheres for cool stars which allow to investigate these issues: the AMES grid based on the new $\mathrm{TiO}$ and $\mathrm{H} 2 \mathrm{O}$ opacities, and the AMES-MT grid which rely on the AMES-TiO and $\mathrm{MT}-\mathrm{H}_{2} \mathrm{O}$ opacities.

We thank David Alexander for helpful discussions and the referee, U.G. Jørgensen, for his very helpful comments. This work was supported in part by grants from CNRS and INSU, NSF grant AST-9720704, NASA ATP grant NAG 5-3018 and LTSA grant NAG 5-3619 to the University of Georgia, and NASA LTSA grant NAG5-3435 to Wichita State University. This work was supported in part by the Pôle Scientifique de Modélisation Numérique at ENS-Lyon. Some of the calculations presented in this paper were performed on the IBM SP2 of CNUSC, the SGI Origin 2000 of the UGA UCNS, and on the 
IBM SP2 of the San Diego Supercomputer Center (SDSC) with support from the National Science Foundation, and on the Cray T3E of the NERSC with support from the DoE. We thank all these institutions for a generous allocation of computer time. 


\section{REFERENCES}

Allard, F. 1990, Model Atmospheres for M-Dwarfs, Ph.D. thesis, University of Heidelberg.

Allard, F., \& Hauschildt, P. H. 1995, ApJ, 445, 433.

Allard, F., Hauschildt, P. H., Miller, S., \& Tennyson, J. 1994, ApJL, 426, 39.

Allard, F., Hauschildt, P. H., Baraffe, I., \& Chabrier, G. 1996, ApJL, 465, L123.

Allard, F., Hauschildt, P. H., Alexander, D. R., \& Starrfield, S. 1997, ARAA, 35, 137.

Alvarez, R., \& Plez, B. 1998, A\&A, 330, 1109.

Aufdenberg, J. P., Hauschildt, P. H., Shore, S. N., \& Baron, E. 1998, ApJ, 498, 837.

Aufdenberg, J. P., Hauschildt, P. H., Sankrit, R., \& Baron, E. 1999, MNRAS, 302, 599.

Baraffe, I., Chabrier, G., Allard, F., \& Hauschildt, P. H. 1995, ApJL, 446, L35.

Baraffe, I., Chabrier, G., Allard, F., \& Hauschildt, P. H. 1997a, A\&A, 327, 1054.

Baraffe, I., Chabrier, G., Allard, F., \& Hauschildt, P. 1997b, A\&A, 327, 1054.

Baraffe, I., Chabrier, G., Allard, F., \& Hauschildt, P. H. 1998a, A\&A, 337, 403.

Baraffe, I., Chabrier, G., Allard, F., \& Hauschildt, P. 1998b, A\&A, 337, 403.

Baron, E., \& Hauschildt, P. H. 1998, ApJ, 495, 370.

Brett, J. M. 1990, A\&A, 231, 440.

Brett, J. M. 1995, A\&A, 295, 736.

Collins, J. G. 1975, J. Phys. B, 0, 304.

Collins, J. G., \& Faÿ, T. D. J. 1974, JQSRT, 14, 1259.

Davis, S., Littleton, J., \& Phillips, J. 1986, ApJ, 309, 449.

Hauschildt, P., Allard, F., Ferguson, J., Baron, E., \& Alexander, D. R. 1999, ApJ, in press.

Hauschildt, P. H., \& Baron, E. 1999, Journal of Computational and Applied Mathematics, $102,41-63$.

Hauschildt, P. H., Baron, E., \& Allard, F. 1997, ApJ, 483, 390. 
Hauschildt, P. H., Allard, F., \& Baron, E. 1999, ApJ, 512, 377.

Hedgecock, L. M., Naulin, C., \& Costes, M. 1995, A\&A, 304, 667.

Irwin, A. W. 1988, A\&A Supp., 74, 145.

Jones, H. R. A., Longmore, A. J., Allard, F., \& Hauschildt, P. H. 1996, MNRAS, 280, 77.

Jørgensen, U. G. 1994, A\&A, 284, 179.

Jørgensen, U. G., Jensen, P., \& Sørensen, G. O. 1994, In Molecular Opacities in the Stellar Environment, P. Thejll and U. Jørgensen, editors, I.A.U. Colloquium 146, page 51, Niels Bohr Institute and Nordita press, Copenhagen.

Kirkpatrick, J. D., Kelly, D. M., Rieke, G. H., Liebert, J., Allard, F., \& Wehrse, R. 1993, ApJ, $402,643$.

Kurucz, R. L. 1993, Molecular data for opacity calculations, Kurucz CD-ROM No. 15.

Langhoff, S. R. 1997, ApJ, 481, 1007.

Leggett, S. K. 1992, ApJS, 82, 351.

Leggett, S. K., Allard, F., Berriman, G., Dahn, C. C., \& Hauschildt, P. H. 1996, ApJS, 104, 117.

Leggett, S. K., Allard, F., \& Hauschildt, P. H. 1998, ApJ, 509, 836.

Leggett, S. K., Allard, F., Dahn, C., Hauschildt, P. H., Kerr, T. H., \& Rayner, J. 1999, ApJ, submitted.

Leinert, C., Allard, F., Richichi, A., , \& Hauschildt, P. H. 1999, A\&A, submitted.

Ludwig, C. B. 1971, Applied Optics, Vol 10, 5, 1057.

Miller, S., Tennyson, J., Jones, H. R. A., \& Longmore, A. J. 1994, In Molecules in the Stellar Environment, U. G. Jørgensen, editor, Lecture Notes in Physics, pages 296-309, Springer-Verlag Berlin Heidelberg.

Partridge, H., \& Schwenke, D. W. 1997, J. Chem. Phys., 106, 4618.

Plez, B. 1992, A\&A Suppl, 94, 527.

Plez, B. 1998, A\&A, 337, 495. 
Schryber, H., Miller, S., \& Tennyson, J. 1995, JQSRT, 53, 373.

Schwenke, D. W. 1998, Chemistry and Physics of Molecules and Grains in Space. Faraday Discussion, 109, 321.

Valenti, J. A., Piskunov, N., \& Johns-Krull, C. M. 1998, ApJ, 498, 851.

This preprint was prepared with the AAS LATEX macros v5.0. 


\section{Tables}

Table 1: $\mathrm{TiO} \mathrm{f}_{\mathrm{el}}$ values $\left(\mathrm{f}_{\mathrm{el}}=f_{v 1 v 2} / q_{v 1 v 2}\right)$

\begin{tabular}{rrrrrrrr}
\hline \hline system & lam0 & B90 & J94 $^{*}$ & DLP86 & L97 & AP98 & adopted \\
\hline alpha & 5170.7 & 0.10 & 0.17 & 0.106 & 0.105 & 0.106 & 0.105 \\
beta & 5605.2 & 0.15 & 0.28 & 0.125 & 0.176 & 0.125 & 0.176 \\
gamma' & 6192.5 & 0.08 & 0.14 & 0.0935 & 0.108 & 0.0935 & 0.108 \\
gamma & 7095.8 & 0.09 & 0.15 & 0.0786 & 0.092 & 0.0786 & 0.092 \\
epsilon & 8407.6 & 0.0024 & 0.014 & $<0.006$ & 0.002 & 0.0023 & 0.002 \\
delta & 8870.9 & 0.02 & 0.048 & - & 0.096 & 0.048 & 0.048 \\
phi & 11044.8 & 0.02 & 0.052 & - & 0.018 & 0.0178 & 0.052 \\
\hline \hline
\end{tabular}

* laboratory values determined by HNC95

B90: Brett (1990)

DLP86: Davis, Littleton, \& Phillips (1986)

J94: Jørgensen (1994)

HNC95: Hedgecock, Naulin, \& Costes (1995)

L97: Langhoff (1997)

AP98: Alvarez \& Plez (1998) 


\section{Figures}

Fig. 1.- Comparison between solar abundance $\mathrm{M}$ dwarf models calculated using AMES$\mathrm{TiO}$ (full curves) and SCAN-TiO (dotted curves) in the blue spectral region. 
Fig. 2.-Comparison between solar abundance $\mathrm{M}$ dwarf models calculated using AMES$\mathrm{TiO}$ (full curves) and SCAN-TiO (dotted curves) in the red spectral region.

Fig. 3.- Comparison between $\mathrm{T}_{\text {eff }}=2900 \mathrm{~K} ; \log (g)=5.0$ and solar abundance models calculated using AMES-TiO (full curves) and SCAN-TiO (dotted curves) based on the same set of TiO oscillator strengths (see "adopted" in Table 1). The models use otherwise identical opacities and parameters. Each model is fully converged, and the synthetic spectra are downgraded to a resolution of $20 \AA$. The positions of the TiO band heads are indicated according to table 8 of Langhoff (1997).

Fig. 4.-Comparison between solar abundance M giant models calculated using AMES$\mathrm{TiO}$ (full curves) and SCAN-TiO (dotted curves) in the blue spectral region.

Fig. 5.- Comparison between solar abundance $\mathrm{M}$ giant models calculated using AMES$\mathrm{TiO}$ (full curves) and SCAN-TiO (dotted curves) in the red spectral region.

Fig. 6. - Comparison between solar abundance $\mathrm{M}$ dwarf models with $T_{\text {eff }}=2500 \mathrm{~K}$ calculated using AMES-TiO and SCAN-TiO. The differences are calculated in the sense AMESTiO minus SCAN-TiO model. The bottom panel gives the results for the Planck-mean (full curve), J-mean (dotted curve), Flux-mean (dashed curve) and Rosseland mean dotdashed curve) opacities.

Fig. 7.-Comparison between solar abundance $\mathrm{M}$ giant models with $T_{\text {eff }}=3000 \mathrm{~K}$ calculated using AMES-TiO and SCAN-TiO. The differences are calculated in the sense AMES$\mathrm{TiO}$ minus SCAN-TiO model. The bottom panel gives the results for the Planck-mean (full curve), J-mean (dotted curve), Flux-mean (dashed curve) and Rosseland mean dotdashed curve) opacities.

Fig. 8.- Comparison between solar abundance $\mathrm{M}$ dwarf models calculated using AMES$\mathrm{H}_{2} \mathrm{O}$ (full curves) and MT- $\mathrm{H}_{2} \mathrm{O}$ (dotted curves) in the near-infrared spectral region. Both sets of models were calculated using AMES-TiO and iterated to convergence with their respective parameters. 
Fig. 9.- Comparison between solar abundance $\mathrm{M}$ dwarf models with $T_{\text {eff }}=2500 \mathrm{~K}$ calculated using AMES- $\mathrm{H}_{2} \mathrm{O}$ and $\mathrm{MT}-\mathrm{H}_{2} \mathrm{O}$. The differences are calculated in the sense AMES- $\mathrm{H}_{2} \mathrm{O}$ minus MT- $\mathrm{H}_{2} \mathrm{O}$. The bottom panel gives the results for the Planck-mean (full curve), J-mean (dotted curve), Flux-mean (dashed curve) and Rosseland mean (dotdashed curve) opacities. Both sets of models were calculated using AMES-TiO and iterated of convergence with their respective parameters.

Fig. 10. - We compare the optical spectral distribution of a NextGen model with $T_{\text {eff }}=$ $3500 \mathrm{~K} ; \log (g)=5.0 ;[\mathrm{M} / \mathrm{H}]=0.0$ (dotted line) with a model converged on the same parameters using the AMES-TiO and $\mathrm{H}_{2} \mathrm{O}$ line lists (full line). The positions of the $\mathrm{TiO}$ band heads are indicated according to table 8 of Langhoff (1997). The region of integration of standard optical broadbands are also shown for reference. The "AMES atmosphere" shows weaker TiO bands, principally due to the smaller oscillator strengths predicted by the Langhoff TiO model.

Fig. 11.- Same as in Figure 10 for the near-infrared portion of the spectrum. The region of integration of the standard near-infrared broadbands are also shown for reference. The "AMES atmosphere" shows stronger $\mathrm{H}_{2} \mathrm{O}$ bands, especially in the trough of the bands, i.e., at $1.6 \mu \mathrm{m}$ and at $2.1 \mu \mathrm{m}$. But little or no changes are seen in the $\mathbf{J}$ bandpass region.

Fig. 12.- The optical Cousins broad-band synthetic photometry of solar metallicity and fixed gravity $(\log (g)=5.0)$ models of the NextGen grid (dotted line), AMES-MT grid (long-dash line), and AMES grid (full line) are compared to the photometric sample of Leggett (1992). This sample contains mostly M dwarfs and metal-depleted M subdwarfs of the solar neighborhood, and becomes scarce in the late-type dwarf regime.

Fig. 13.- Same as Fig. 12 for near-infrared broad-band colors covering the water opacity range. Please note that the hot star tail of the sample, near $\mathrm{H}-\mathrm{K}=0.1$, is reproduced by the NextGen and AMES-MT models for the lower gravities predicted by evolution models for 5 to 10 Gyrs isochrones. Of the models shown, only the NextGen are grain-less, which explains their curling up at the low temperature end compared to AMES-MT models.

Fig. 14.- Same as Fig. 12 and 13 for broad-band colors sampling side-to-side of the SED's flux peak. 